\title{
Isomorphism of a Piecewise Linear Transformation to a Markov Automorphism
}

\author{
By
}

Shunji ITO* and Makoto MORI**

D. Ornstein and others proved important isomorphism theorems for a large class of automorphisms, i.e. measure preserving transformations. However, it is also interesting to explicitely determine an isomorphic mapping in each concrete case. R. Adler and B. Weiss [1] explicitely constructed an isomorphism between an ergodic group automorphism of a 2-dimensional torus and a Markov automorphism. Y. Takahashi [8] gave an isomorphism between a $\beta$-automorphism and a Markov automorphism. The crucial point of their argument lies in the fact that the metrical entropy coincides with the topological entropy for these automorphisms. Using this fact and Parry's result [3], they showed that the representation mapping of the automorphism in consideration to a Markov subshift (of a symbolic dynamics) is actually an isomorphism in each case.

In this paper, we will explicitely construct an isomorphism of a piecewise linear transformation (a generalization of $\beta$-automorphism) to a Markov automorphism. Since the metrical entropy of such a transformation does not always attain its topological entropy, we cannot use the method mentioned above, so instead of topological entropy we use free energy as our main tool.

In $\$ 1$ we define the free energy of a Markov subshift and show under certain conditions that a shift invariant measure with the minimal free energy is unique. This is a generalization of Parry's result about topological entropy [3]. In $\S 2$ we define a piecewise linear transformation and investigate its properties. In $\S 3$ we construct an isomorphic mapping from a piecewise linear transformation to a Markov automor-

Communicated by H. Araki, February 3, 1975.

* Department of Mathematics, Tsuda College, Kodaira 187, Japan.

** Department of Mathematics, University of Tsukuba, Ibaraki 300-31, Japan. 
phism.

We would like to express our hearty thanks to Professors Haruo Totoki, Yuji Ito and Yoichiro Takahashi for their valuable advices.

\section{§ 1. Markov Potential Function and Its Free Energy}

Let $I^{z}$ be an infinite product of state space $I$, where $I=\{0,1, \cdots, s\}$ or $\{0,1,2, \cdots\}$ and $z=\{\cdots,-1,0,1, \cdots\}$. Let $\omega(n)$ be the $n$-th coordinate of $\omega$ in $I^{z}$ and let $\sigma$ be a shift operator on $I^{z}$ such that

$$
(\sigma \omega)(n)=\omega(n+1) .
$$

Definition 1-1. A function $U: I^{\boldsymbol{z}} \rightarrow[0, \infty]$ is called a (simple) Markov potential function, if $U(\omega)=U(\omega(0), \omega(1))$ for all $\omega \in I^{z}$.

Definition 1-2. A Markov potential function $U$ is called irreducible, if, for any $i, j \in I$, there exists a finite sequence $\left(i_{0}, i_{1}, \cdots, i_{n}\right)$ such that $i_{0}=i, i_{n}=j$, and $U\left(i_{k}, i_{k+1}\right)<\infty$ for all $0 \leqq k \leqq n-1$.

Definition 1-3. A Markov potential function $U$ is called of PerronFrobenius type if

(1) $U$ is irreducible

(2) $Q(i, j)$ defined by $Q(i, j)=e^{-U(i, j)}$

has positive right and left eigenvectors, $r=\left(r_{i}\right)$ and $l=\left(l_{i}\right)$ respectively, with a common positive eigenvalue $\lambda$, such that

$$
\begin{aligned}
& \sum_{j \in I} Q(i, j) r_{j}=\lambda r_{i} \quad \text { for all } i \in I, \\
& \sum_{i \in I} Q(i, j) l_{i}=\lambda l_{j} \quad \text { for all } j \in I, \\
& \sum_{i \in I} l_{i} r_{i}<\infty .
\end{aligned}
$$

Definition 1-4. Let $\lambda, r=\left(r_{i}\right)$ and $l=\left(l_{i}\right)$ be the ones defined by Perron-Frobenius type potential $U$ as above. The Gibbs measure $\nu=\nu(U)$ is an ergodic $\sigma$-invariant Markov measure with initial distribution

$$
\pi_{i}=l_{i} r_{i} /\left(\sum_{j \in I} l_{j} r_{j}\right) \quad \text { for } \quad i \in I
$$


and transition probabilities

$$
P_{i j}=\frac{r_{j} Q(i, j)}{\lambda r_{i}} \text { for } i, j \in I \text {. }
$$

When $I=\{0,1, \cdots, s\} \quad(s<\infty)$, free energy $f_{U}(\mu)$ is defined by (cf. Spitzer [7]), $f_{U}(\mu)=\int U d \mu-h_{\mu}(\sigma)$, where $\mu$ is a $\sigma$-invariant probability measure on $\left(I^{z}, \sigma\right)$. Now we extend this definition.

Definition 1-5. Let $I=\{0,1,2, \cdots\}$ and let $\mu$ be a $\sigma$-invariant probability measure on $\left(I^{z}, \sigma\right)$ with finite metrical entropy $h_{\mu}(\sigma)$. Then free energy $f_{U}(\mu)$ is defined by,

$$
f_{U}(\mu)=\varlimsup_{m \rightarrow \infty} \varlimsup_{n \rightarrow \infty} \sum_{x \in I_{m^{n}}} \mu([x]) U_{n}^{m}(x)-h_{\mu}(\sigma),
$$

where $I_{m}=\left\{0,1, \cdots, m, m^{*}\right\}, m^{*}=\{m+1, m+2, \cdots\},[x]$ is the cylinder set generated by $x \in I_{m}{ }^{n}$ and $U_{n}{ }^{n}$, a function on $I_{m}{ }^{n}$, is defined in the following way. For $x, y \in I_{m}{ }^{\prime}=\{0,1, \cdots, m\}, k \geqq 1$, we put

$$
\begin{aligned}
& U_{2}{ }^{m}(x, y)=U(x, y), \\
& U_{k+1}^{m}(x, \underbrace{m^{*}, \cdots, m^{*}}_{k}) \\
& =-\frac{1}{k} \log \sum_{\substack{i_{1} \geqq m+1 \\
\text { and } \\
i_{k} \geqq m+1}} r_{i_{k}} \exp \left[-U\left(x, i_{1}\right)-\sum_{j=1}^{k-1} U\left(i_{j}, i_{j+1}\right)\right] \\
& U_{k+2}^{m}(x, \underbrace{m^{*}, \cdots, m^{*}}_{k}, y) \\
& =-\frac{1}{k+1} \log \sum_{\substack{i_{1} \geq m+1 \\
\text { and } \\
i_{k} \geqq m+1}} \exp \left[-U\left(x, i_{1}\right)-\sum_{j=1}^{k-1} U\left(i_{j}, i_{j+1}\right)-U\left(i_{k}, y\right)\right] \\
& U_{k+1}^{m}(\underbrace{m^{*}, \cdots, m^{*}}_{k+1}) \\
& =-\frac{1}{k} \log _{\substack{i_{1} \geq m+1 \\
i_{k+1} \geq m+1}} \frac{l_{i_{1}} r_{i_{k+1}}}{\sum_{j} l_{j} r_{j}} \exp \left[-\sum_{j=1}^{k} U\left(i_{j}, i_{j+1}\right)\right],
\end{aligned}
$$

and

$$
\begin{aligned}
& U_{k+1}^{m}(\underbrace{m^{*}, \cdots, m^{*}}_{k}, y) \\
& \quad=-\frac{1}{k} \log _{\substack{i_{1} \geq m+1 \\
i_{k} \geqq m+1}} \frac{l_{i_{1}}}{\sum_{j} l_{j} r_{j}} \exp \left[-\sum_{j=1}^{k-1} U\left(i_{j}, i_{j+1}\right)-U\left(i_{k}, y\right)\right]
\end{aligned}
$$


(we define $\log 0=-\infty$ ). Moreover we define $U_{n}{ }^{m}$ inductively by

$$
\begin{aligned}
& (n-1) U_{n}^{m}\left(x_{1}, \cdots, x_{n}\right) \\
& \quad=(i-1) U_{i}^{m}\left(x_{1}, \cdots, x_{i}\right)+(n-i) U_{n-i+1}^{m}\left(x_{i}, \cdots, x_{n}\right)
\end{aligned}
$$

if $x_{j} \in I_{m}(1 \leqq j \leqq n)$ and $x_{i} \in I_{m}{ }^{\prime}$.

Remark 1. For $x_{i} \in I_{m}{ }^{\prime}(1 \leqq i \leqq n)$,

$$
(n-1) U_{n}^{m}\left(x_{1}, \cdots, x_{n}\right)=\sum_{i=1}^{n-1} U\left(x_{i}, x_{i+1}\right) \text {. }
$$

We get, for $x \in I_{m}{ }^{n}$,

$$
\begin{aligned}
& U_{n}^{m}(x)=-\log \lambda-\frac{1}{n-1} \log P_{n}{ }^{m}(x)-\frac{1}{n-1} \log r_{x_{1}}^{m}+\frac{1}{n-1} \log r_{x_{n}}^{m} \\
& \text { (including } \infty=\infty \text { ) }
\end{aligned}
$$

where $r_{i}{ }^{m}=r_{i}$, $i \in I_{m}{ }^{\prime}, r_{m^{*}}^{m}=1$, and $P_{n}{ }^{m}(x)$, a function on $I_{m}{ }^{n}$, is defined by the following; for $x, y \in I_{m}{ }^{\prime}, k \geqq 1$

$$
\begin{aligned}
& P_{2}^{m}(x, y)=P_{x, y} \\
& P_{k+1}^{m}(x, \underbrace{m^{*}, \cdots, m^{*}}_{k})=\sum_{\substack{i_{1} \geq m+1 \\
i_{k} \geq m+1}} P_{x, i_{1}} P_{i_{1}, i_{2}} \cdots P_{i_{k-1}, i_{k}} \\
& P_{k+2}^{m}(\underbrace{m^{*}, \cdots, m^{*}}_{k}, y)=\sum_{\substack{i_{1} \geq m+1 \\
i_{k} \geq m+1}} P_{x, i_{1}} P_{i_{1}, i_{2}} \cdots P_{i_{k-1}, i_{k}} P_{i_{k}, y} \\
& P_{k+1}^{m} \underbrace{m^{*}, \cdots, m^{*}}_{k+1})=\underbrace{}_{\substack{i_{1} \geq m+1 \\
i_{k+1} \geq m+1}} \pi_{i_{1}} P_{i_{1}, i_{2}} \cdots P_{i_{k}, i_{k+1}} \\
& P_{k+1}^{m} \underbrace{m^{*}, \cdots, m^{*}}, y)=\sum_{\substack{i_{1} \geq m+1 \\
i_{k} \geq m+1}} \pi_{i_{1}} P_{i_{1}, i_{2}} \cdots P_{i_{k-1}, i_{k}} P_{i_{k}, y}
\end{aligned}
$$

and

$$
P_{n}^{m}\left(x_{1}, \cdots, x_{n}\right)=P_{i}^{m}\left(x_{1}, \cdots, x_{i}\right) P_{n-i+1}^{m}\left(x_{i}, \cdots, x_{n}\right)
$$

where $x_{j} \in I_{m} \quad(1 \leqq j \leqq n), x_{i} \in I_{m}{ }^{\prime}$.

Remark. If $x_{i} \in I_{n}{ }^{\prime}(1 \leqq j \leqq n)$,

$$
P_{n}{ }^{m}\left(x_{1}, \cdots, x_{n}\right)=\prod_{i=1}^{n-1} P_{x_{i}, x_{i+1}} .
$$


Theorem 1. Let $U$ be a Markov potential of Perron-Frobenius type on $\left(I^{z}, \sigma\right)$, and let $\lambda$ be an eigenvalue of $Q=\exp (-U)$ as in Definition 1-3. Then, for any $\sigma$-invariant probability measure $\mu$ with finite entropy, we have

$$
f_{U}(\mu) \geqq-\log \lambda
$$

In particular, (1.1) holds with equality if and only if $\mu=\nu$ and $h_{\nu}(\sigma)<\infty$, where $\nu=\nu(U)$ is the Gibbs measure defined by $U$.

Proof. 1st stage: We will show (1.1) holds with equality if $\mu=\nu$ and $h_{\nu}(\sigma)<\infty$. We denote by $q_{m}$ the $\sigma$-algebra generated by the cylinder sets of $I_{m} z$, and by $h_{\mu}\left(\sigma \mid \mathscr{g}_{m}\right)$ we mean the entropy of the factor of $\sigma$ on $q_{m}$. Evidently,

$$
\begin{aligned}
h_{\mu}(\sigma) & =\lim _{m \rightarrow \infty} h_{\mu}\left(\sigma \mid g_{m}\right) \\
& =\lim _{m \rightarrow \infty} \lim _{n \rightarrow \infty} \frac{1}{n} \sum_{x \in I_{m^{n}}} \mu([x]) \log \mu([x]) .
\end{aligned}
$$

Therefore,

$$
\begin{aligned}
f_{U}(\nu)= & \varlimsup_{m \rightarrow \infty} \varlimsup_{n \rightarrow \infty}\left[-\log \lambda-\frac{1}{n-1} \sum_{x \in I_{m}{ }^{n}} \nu([x]) \log \nu([x])\right. \\
& \left.+\frac{1}{n-1} \sum_{i \in I_{m}} \pi_{i} \log \pi_{i}\right]+\lim _{m \rightarrow \infty} \lim _{n \rightarrow \infty} \frac{1}{n} \sum_{x \in I_{m}{ }^{n}} \nu([x]) \log \nu([x]) \\
= & -\log \lambda .
\end{aligned}
$$

2nd stage: Let $\mu$ be a $\sigma$-invariant probability measure with finite entropy which does not equal to $\nu$. Let $\left\{\tilde{\pi}_{i}\right\}$ be a distribution such that

$$
\begin{aligned}
& \text { (1) } \sum_{i \in I} \tilde{\pi}_{i}=1 \\
& \text { (2) }-\sum_{i \in I} \mu([i]) \log \tilde{\pi}_{i}<\infty \\
& \text { (3) } \tilde{\pi}_{i}>0 \text { if and only if } \pi_{i}>0 .
\end{aligned}
$$

We define $\widetilde{\nu}$ the Markov measure with initial distribution $\widetilde{\pi}_{i}$ and transition probability $P_{i j}$. (Notice that $\tilde{\nu}$ is not always $\sigma$-invariant.) Then it is easy to see that $\widetilde{\nu} \sim \nu$. From the $\sigma$-invariance of $\mu$, we get

$$
\frac{1}{n-1} \sum_{x \in I_{m}{ }^{n}} \mu([x]) \log P_{n}{ }^{m}(x) \leqq \sum_{x, y \in I_{m^{\prime}}} \mu([x, y]) \log P_{x, y} .
$$


Therefore,

$$
\varlimsup_{n \rightarrow \infty} \frac{1}{n-1} \sum_{x \in I_{m^{n}}} \mu([x]) \log P_{n}^{m}(x) \leqq \sum_{x, y \in I^{\prime} m} \mu([x, y]) \log P_{x, y} .
$$

If $\lim _{m \rightarrow \infty} \sum_{x, y \in I^{\prime} m} \mu([x, y]) \log P_{x, y}=-\infty$, then

$$
f_{U}(\mu)=\varlimsup_{m \rightarrow \infty} \varlimsup_{n \rightarrow \infty} \sum_{x \in I_{m} n} \mu([x]) U_{n}^{m}(x)-h_{\mu}(\sigma)=\infty>-\log \lambda .
$$

If $\lim _{m \rightarrow \infty} \sum_{x, y \in I_{m^{\prime}}} \mu([x, y]) \log P_{x, y}>-\infty$, then

$$
\begin{aligned}
\varlimsup_{m \rightarrow \infty} & \varlimsup_{n \rightarrow \infty} \frac{1}{n-1} \sum_{x \in I_{m}{ }^{n}} \mu([x]) \log P_{n}^{m}(x) \\
& \leqq \sum_{x, y \in I} \mu([x, y]) \log P_{x, y} \\
& =\frac{1}{n-1} \sum_{x=\left(x_{1}, \cdots, x_{n}\right) \in I^{n}} \mu([x]) \log P_{x_{1}, x_{2}} P_{x_{2}, x_{3}} \cdots P_{x_{n-1}, x_{n}} \\
& =\frac{1}{n-1} \sum_{x \in I^{n}} \mu([x]) \log \widetilde{\nu}([x])-\frac{1}{n-1} \sum_{i \in I} \mu([i]) \log \tilde{\pi}_{i} \\
& \leqq \varlimsup_{m \rightarrow \infty} \frac{1}{n-1} \sum_{x \in I_{m^{n}}} \mu([x]) \log \widetilde{\nu}([x])-\frac{1}{n-1} \sum_{i \in I} \mu([i]) \log \tilde{\pi}_{i} .
\end{aligned}
$$

Thus,

$$
\begin{aligned}
f_{U}(\mu)= & \varlimsup_{m \rightarrow \infty} \varlimsup_{n \rightarrow \infty} \sum_{x \in I_{m}{ }^{n}} \mu([x]) U_{n}{ }^{m}(x)-\lim _{m \rightarrow \infty} h_{\mu}\left(\sigma \mid \mathscr{F}_{m}\right) \\
\geqq & -\log \lambda-\varlimsup_{m \rightarrow \infty} \frac{1}{n-1} \sum_{x \in I_{m}{ }^{n}} \mu([x]) \log \tilde{\nu}([x]) \\
& +\frac{1}{n-1} \sum_{i \in I} \mu([i]) \log \tilde{\pi}_{i}+\lim _{m \rightarrow \infty} \frac{1}{n} \sum_{x \in I_{m}{ }^{n}} \mu([x]) \log \mu([x]) \\
\geqq & \frac{1}{n-1}\left[\lim _{m \rightarrow \infty} \sum_{x \in I_{m} n} \mu([x]) \log \left(\frac{\mu([x])}{\widetilde{\mathcal{\nu}}([x])}\right)+\sum_{i \in I} \mu([i]) \log \tilde{\pi}_{i}\right] \\
& -\log \lambda .
\end{aligned}
$$

On the other hand, since $\nu$ is ergodic and $\mu$ is $\sigma$-invariant, it follows $\mu \chi \nu$. We cite the followings.

\section{Lemma 1 ([5]).}

$$
\lim _{m \rightarrow \infty} \sum_{x \in I_{m^{n}}} \mu([x]) \log \frac{\mu([x])}{\widetilde{\mathcal{V}}([x])} \geqq \sum_{x \in I^{n}} \mu([x]) \log \frac{\mu([x])}{\widetilde{\mathcal{V}}([x])} .
$$


Lemma 2. (Dobrushin, Gelfand, Yaglom and Perez [5] (p. 20)). If $\mu_{1}$ and $\mu_{2}$ are probability measures on $I^{z}$ and $\mu_{1} \nless \mu_{2}$, then

$$
\sum_{x \in I n} \mu_{1}([x]) \log \left(\frac{\mu_{1}([x])}{\mu_{2}([x])}\right) \rightarrow \infty \text { as } n \rightarrow \infty
$$

By the above lemmas, we can take $n \geqq 1$ such that

$$
\sum_{i \in I} \mu([i]) \log \tilde{\pi}_{i}+\sum_{x \in I^{n}} \mu([x]) \log \left(\frac{\mu([x])}{\widetilde{\nu}([x])}\right)>0,
$$

and thus we can conclude that

$$
f_{U}(\mu)>-\log \lambda \text {. }
$$

Remark 1-6. If a Markov potential $U$ satisfies the condition in Theorem 1 and takes values only 0 and $\infty$, then $Q=e^{-U}$ becomes a structure matrix of a Markov subshift [2]. Hence inf $-f_{U}(\mu)=-f_{U}(\nu)$ and we see that $\nu$ is the maximal Markov measure [3].

Let $U$ be a Markov potential on $\left(I^{z}, \sigma\right)$ which satisfies the conditions in Theorem 1 and let $\nu$ be the Gibbs measure defined by $U$.

Main Lemma. Let $(X, T, \mu)$ be an automorphism with finite entropy. Then $(X, T, \mu)$ is isomorphic to $\left(I^{z}, \sigma, \nu\right)$, if there exist a 1-1 ( $\mu$-a.e.) bi-Borel mapping $\varphi: X \rightarrow I^{z}$, $\mu$-integrable functions $V\left(\omega^{\prime}\right)$ and $h\left(\omega^{\prime}\right) \quad\left(\omega^{\prime} \in X\right)$ such that

(1) $\sigma \cdot \varphi=\varphi \cdot T, \quad \mu$-a.e.

(2) $U\left(\varphi\left(\omega^{\prime}\right)\right)=V\left(\omega^{\prime}\right)+h\left(\omega^{\prime}\right), \quad \mu$-a.e.

$$
\int h\left(\omega^{\prime}\right) d \mu\left(\omega^{\prime}\right)=0,
$$

(3) $f_{U}(\nu)=\int d \mu\left(\omega^{\prime}\right) V\left(\omega^{\prime}\right)-h_{\mu}(T)$,

(4) $\lim _{m \rightarrow \infty} \lim _{n \rightarrow \infty} \sum_{x \in I_{m}^{n}} \mu \cdot \varphi^{-1}([x]) U_{n}^{m}(x)=\int d \mu \cdot \varphi^{-1} U$.

Proof. Let $\mu \cdot \varphi^{-1}=\mu^{\prime}$, then from the condition (1) $\mu^{\prime}$ is a $\sigma$-invariant measure with finite entropy $h_{\mu}(T)$. Therefore to show that they are isomorphic it is sufficient to prove that $f_{U}\left(\mu^{\prime}\right)=f_{U}(\nu)$. 


$$
\begin{aligned}
f_{U}\left(\mu^{\prime}\right) & =\varlimsup_{m \rightarrow \infty} \varlimsup_{n \rightarrow \infty} \sum \mu^{\prime}([x]) U_{n}^{m}(x)-h_{\mu^{\prime}}(\sigma) \\
& =\int d \mu\left(\omega^{\prime}\right) U\left(\varphi\left(\omega^{\prime}\right)\right)-h_{\mu}(T) \\
& =\int d \mu\left(\omega^{\prime}\right) V\left(\omega^{\prime}\right)-h_{\mu}(T) \\
& =f_{U}(\nu) .
\end{aligned}
$$

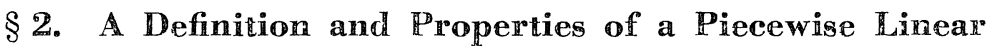

\section{Transformation}

We define a piecewise linear transformation according to [6].

Let $\beta=\left(\beta_{0}, \beta_{1}, \cdots, \beta_{N}\right)$ is an $N+1$-tuple vector such that $\beta_{k}>1$ for $0 \leqq k \leqq N$ and $\sum_{k=0}^{N} \beta_{k}{ }^{-1} \geqq 1>\sum_{k=0}^{N-1} \beta_{k}^{-1}$. A partition $\left\{A_{i}\right\}_{i=0, \ldots, N}$ of interval $[0,1)$ is defined by

$$
\begin{aligned}
& A_{0}=\left[0, \beta_{0}^{-1}\right) \\
& A_{i}=\left(\sum_{k=0}^{i-1} \beta_{k}^{-1}, \sum_{k=0}^{i} \beta_{k}^{-1}\right) \quad i=1, \cdots, N-1 . \\
& A_{N}=\left(\sum_{k=0}^{N-1} \beta_{k}^{-1}, 1\right) .
\end{aligned}
$$

We define mappings $T:[0,1) \rightarrow[0,1)$ and $\pi ;[0,1) \rightarrow S^{\mathbf{N}}$ by

$$
T x=\beta_{i}\left(x-\sum_{k=0}^{i-1} \beta_{k}{ }^{-1}\right) \quad \text { if } \quad x \in A_{i}, i=0,1, \cdots, N,
$$

and

$$
\pi(x)=\left(a_{0}(x), a_{1}(x), \cdots, a_{n}(x), \cdots\right),
$$

$a_{n}(x)=i$ if $T^{n} x \in A_{i}$, respectively, where $S=\{0,1, \cdots, N\}$ and $S^{N}$ is its one-sided product. Then the mapping $T$ can be realized as the shift $\sigma$ on $S^{\mathbf{N}}$ by the mapping $\pi$, namely

$$
\pi \cdot T=\sigma \cdot \pi
$$

For convenience we define a sequence corresponding to 1 by

$$
\begin{aligned}
\pi(1) & =\left(a_{0}(1), a_{1}(1), \cdots, a_{n}(1), \cdots\right) \\
& =\sup _{x \in[0,1)}\left(a_{0}(x), a_{1}(x), \cdots, a_{n}(x), \cdots\right)
\end{aligned}
$$


where supremum is taken over with respect to the lexicographical order. Then it is easy to see that for $x \in[0,1)$

$$
\begin{aligned}
x= & \left.\sum_{k=0}^{a_{0}(x)-1} \beta_{k}^{-1}+\beta_{a_{0}(x)}^{-1} \sum_{k=0}^{a_{1}(x)-1} \beta_{k}^{-1}\right)+\cdots \\
& +\beta_{a_{0}(x)}^{-1} \cdots \beta_{a_{n}(x)}^{-1}\left(\sum_{k=0}^{a_{n+1}(x)-1} \beta_{k}^{-1}\right)+\cdots
\end{aligned}
$$

We put

$$
T^{j} 1=\sum_{k=0}^{a_{j(1)}-1} \beta_{k}^{-1}+\beta_{a_{j}(1)}^{-1}\left(\sum_{k=0}^{a_{j+1}(1)-1} \beta_{k}^{-1}\right)+\cdots
$$

We already know the results that the endomorphism $T$ has an invariant probability measure $\nu_{\beta}{ }^{+}$equivalent to Lebesgue measure, and its unnormalized density $f(x)$ is given by

$$
f(x)=\sum_{n=0}^{\infty} \frac{X_{\left[0, T^{n} 1\right)}}{\beta_{a_{0}(1)} \beta_{a_{1}(1)} \cdots \beta_{a_{n}(1)}}(x),
$$

where $X_{A}$ is the characteristic function of $A[6]$.

Let $\pi[0,1)=X_{\beta}{ }^{+} \subset S^{\boldsymbol{N}}$ and let $\mu_{\beta}{ }^{+}$be an induced measure of $\nu_{\beta}{ }^{+}$by $\pi$. Then $\left[X_{\beta}{ }^{+}, \sigma, \mu_{\beta}{ }^{+}\right]$becomes an endomorphism which is homeomorphic to $\left([0,1), T, \nu_{\beta}^{+}\right)$. We call this endomorphism a piecewise linear endomorphism.

\section{Proposition 2. 1.}

(1) For any $\omega \in X_{\beta}$

$$
\sigma^{n}(1)\left(a_{0}(1), a_{1}(1), \cdots, a_{n}(1), \cdots\right), \text { for } n \geqq 0 .
$$

(2) Let $X_{\beta}^{n}=\left\{\left[a_{0}(x), \cdots, a_{n}(x)\right]: x \in[0,1)\right\}$, then

$$
\max \left\{\left[b_{0}, \cdots, b_{n}\right] \in X_{\beta}^{n}\right\}=\left[a_{0}(1), \cdots, a_{n}(1)\right] .
$$

The proofs of this proposition are very easy and we omitt it (c.f. [6]).

The piecewise linear transformation $\left(X_{\beta}, \sigma, \mu_{\beta}\right)$ which is the natural extension of $\left(X_{\beta}{ }^{+}, \sigma, \mu_{\beta}{ }^{+}\right)$is defined by

$$
\begin{aligned}
& X_{\beta}=\left\{\omega \in S^{z} ;(\omega(n), \omega(n+1), \cdots) \in X_{\beta}{ }^{+}, \text {for all } n\right\}, \\
& \iota_{\beta}\left(\left[b_{0}, \cdots, b_{n}\right]\right)=\mu_{\beta}{ }^{+}\left(\left[b_{0}, \cdots, b_{n}\right]\right),
\end{aligned}
$$


where $\left[b_{0}, \cdots, b_{n}\right] \in X_{\beta}^{n}$. Note that $\mu_{\beta}$ is well-defined because of $\sigma$-invariance of $\mu_{\beta}^{+}$.

\section{§ 3. Isomorphism of a Piecewise Linear Transformation to a Markov Automorphism}

In this section we will prove the following Theorem 2 by appealing to Main Lemma in Section 1 and a mapping $\varphi$ which is similar to the mapping introduced by Y. Takahashi for $\beta$-transformation ([8]).

Theorem 2. A piecewise linear transformation $\left(X_{\beta}, \sigma, \mu_{\beta}\right)$ is isomorplic to a Markov automorphism.

To prove this Theorem we begin with the construction of a mapping $\varphi: X_{\beta} \rightarrow I^{z}$ where $I=\{-N+1, \cdots,-1,0,1, \cdots\}$.

For $\omega \in X_{\beta}, \tau(\omega)$ is defined by

$$
\tau(\omega)=\left\{\begin{array}{l}
\sup \left\{i: i \geqq 1, \omega \in B_{i}\right\}, \\
0, \quad \text { if } \quad \omega \in X_{\beta} \backslash \bigcup_{i \geqq 1} B_{i},
\end{array}\right.
$$

where $B_{i}=\left\{\omega \in X_{\beta}:(\omega(-i), \cdots, \omega(-1))=\left(a_{0}(1), \cdots, a_{i-1}(1)\right)\right\}$.

Let $C_{i}=\left\{\omega \in X_{\beta}: \tau(\omega)=i\right\}$, for $i=1,2, \cdots$,

$$
\begin{aligned}
& =\left\{\omega \in X_{\beta}: \tau(\omega)=0, \omega(-1)=-i\right\}, \text { for }-N+1 \leqq i \leqq 0, \\
C_{\infty} & \left.=\omega \in X_{\beta}: \tau(\omega)=\infty\right\} .
\end{aligned}
$$

It is easy to see that $\mu_{\beta}\left(C_{\infty}\right)=0$ and $\sigma C_{\infty}=C_{\infty}$. Define a mapping $\varphi: X_{\beta} \backslash C_{\infty} \rightarrow I^{z}$ by

$$
(\varphi(\omega))(n)=i \quad \text { if } \quad \omega \in \sigma^{n} C_{i}, n \in \boldsymbol{Z}, i \in I .
$$

Then, it is easy to see that the mapping $\varphi$ is a Borel injection and satisfies

$$
\varphi \cdot \sigma=\sigma^{-1} \cdot \varphi
$$

Lemma 3-1. (1) If $\omega \in C_{i}, 1 \leqq i \leqq \infty$, then

$$
\sigma^{-1} \omega \in C_{i-1} \text {. }
$$


(2) If $\omega \in C_{i}, i \leqq 0$, and $\sigma^{-1} \omega \in C_{j}, j \geqq 1$, then

$$
a_{j}(1)>-i=|i| \text {. }
$$

Proof. (1) Suppose $\sigma^{-1}\left(\omega \in C_{j} j \geqq i\right.$, then

$$
(\omega(-j-1), \cdots, \omega(-1))<\left(a_{0}(1), \cdots, a_{j}(1)\right)
$$

and

$$
(\omega(-j-1), \cdots, \omega(-2))=\left(a_{0}(1), \cdots, a_{j-1}(1)\right) .
$$

Hence

$$
\text { (1) }(-1)<a_{j}(1)
$$

and so

$$
(\omega(-i), \cdots, \omega(-1))<\left(\omega(-i), \cdots, \omega(-2), a_{j}(1)\right) .
$$

This contradicts to the maximality of $(\omega(-i), \cdots, \omega(-1))$.

(2) By the assumption, the $j$-tuple

$$
(\omega(-j-1), \cdots, \omega(-2))=\left(a_{0}(1), \cdots, a_{j-1}(1)\right)
$$

is maximum. From $\omega \in C_{i},(i \leqq 0)$, it follows that $\omega(-1)=-i=|i|$ and

$$
(\omega(-j-1), \cdots, \omega(-1))<\left(a_{0}(1), \cdots, a_{j}(1)\right) .
$$

Therefore

$$
a_{j}(1)>-i=|i| \text {. }
$$

We define a Markov potential $U$ on $I^{z}$ by

$$
U(i, j)=\left\{\begin{array}{l}
\log \beta_{-i}, \quad-N+1 \leqq i \leqq 0, \quad-N+1 \leqq j \leqq 0, \\
\log \beta_{N}, \quad i=1, \quad-N+1 \leqq j \leqq 0, \\
\log \beta_{a_{i-1}(1)}, \quad i \geqq 2, \quad j=i-1, \\
\log \beta_{-i}, \quad-N+1 \leqq i \leqq 0, \quad j>0 \quad \text { and } \quad a_{j}(1)>-i, \\
\infty, \quad \text { otherwise. }
\end{array}\right.
$$

It is easy to see that this potential $U$ is irreducible. We show that $U$ is of the Perron-Frobenius type by finding an eigenvalue $\lambda$ of $Q=e^{-U}$ and corresponding eigenvectors to $\lambda$. 
Put $\lambda, l=\left(l_{i}\right)_{i \geq-N+1}$ and $r=\left(r_{i}\right)_{i \geq-N+1}$ as follows,

$$
\begin{aligned}
\lambda & =1, \\
l_{i} & =1, \quad-N+1 \leqq i \leqq 0, \\
& =T^{i} 1, \quad i \geqq 1, \\
r_{i} & =\beta_{a_{0}(1)}^{-1} \beta_{a_{1}(1)}^{-1} \cdots \beta_{a_{i-1}(1)}^{-1}, \quad i \geqq 1, \\
& =\beta_{-i}^{-1}\left(1+\sum_{j \geqq 1} M_{i j} \beta_{a_{0}(1)}^{-1} \cdots \beta_{a_{j-1}(1)}^{-1}\right), \quad-N+1 \leqq i \leqq 0,
\end{aligned}
$$

where

$$
\begin{aligned}
M_{i j} & =1, \quad \text { if } \quad Q(i, j)>0, \\
& =0, \quad \text { if } \quad Q(i, j)=0 .
\end{aligned}
$$

Then it is easy to see that

$$
\begin{aligned}
& \sum_{i \in I} l_{i} Q(i, j)=l_{j}=\lambda l_{j}, \quad \text { for all } j, \\
& \sum_{j \in I} Q(i, j) r_{j}=r_{i}, \quad \text { for all } i, \\
& \sum_{i \in I} r_{i} l_{i}<\infty .
\end{aligned}
$$

Therefore $U$ is of the Perron-Frobenius type. Let $\nu$ be the Gibbs measure corresponding to $U$. From the fact $-\sum \pi_{i} \log \pi_{i}<\infty$,

$$
h_{\nu}(\sigma)<\infty \text {. }
$$

Hence Theorem 1 in Section 1 implies

$$
f_{U}(\nu)=-\log 1=0 .
$$

Now we will define $V\left(\omega^{\prime}\right)$ on $\left(X_{\beta}, \sigma, \mu_{\beta}\right)$ to apply the Main Lemma. Let

$$
V\left(\omega^{\prime}\right)=\sum_{i \in S} X_{i}\left(\omega^{\prime}\right) \log \beta_{i}
$$

where $X_{i}\left(\omega^{\prime}\right)$ is the characteristic function of $\left\{\omega^{\prime}: \omega^{\prime}(-1)=i\right\}$. Then

$$
\begin{aligned}
\int d \mu_{\beta}\left(\omega^{\prime}\right) V\left(\omega^{\prime}\right) & =\int d \mu_{\beta}\left(\omega^{\prime}\right) V\left(\sigma^{-1} \omega^{\prime}\right) \\
& =\int \sum_{i \in S} X_{\left[\omega^{\prime}: \omega^{\prime}(0)=i\right]}\left(\omega^{\prime}\right) \log \beta_{i} d \mu_{\beta}\left(\omega^{\prime}\right) .
\end{aligned}
$$

On the other hand by [4], 


$$
h_{\mu_{\beta}}(\sigma)=\int d \mu_{\beta} \log \phi^{\prime}\left(\omega^{\prime}\right)
$$

where $\phi^{\prime}\left(\omega^{\prime}\right)$ is the derivative of the graph of $\phi$-expansion. Hence

$$
\int d \mu_{\beta}\left(\omega^{\prime}\right) V\left(\omega^{\prime}\right)-h_{\mu_{\beta}}(\sigma)=0=f_{U}(\nu) .
$$

We have already proved $\varphi$ satisfies the conditions (1) and (3) in Main Lemma, and hence it suffices to show only the conditions (2) and (4).

1) In case $\omega^{\prime} \in C_{i}, 2 \leqq i<\infty$, it holds $\omega^{\prime}(-1)=a_{i-1}(1)$ and hence

$$
V\left(\omega^{\prime}\right)=\log \beta_{a_{i-1}(1)} .
$$

On the other hand by Lemma $3-1$, it follows

$$
\left(\varphi\left(\omega^{\prime}\right)\right)(0)=i, \quad\left(\varphi\left(\omega^{\prime}\right)\right)(1)=i-1 .
$$

Thus

$$
\begin{aligned}
U\left(\varphi\left(\omega^{\prime}\right)\right) & =U\left(\left(\varphi\left(\omega^{\prime}\right)\right)(0),\left(\varphi\left(\omega^{\prime}\right)\right)(1)\right) . \\
& =\log \beta_{a_{i-1}(1)} .
\end{aligned}
$$

2) In case $\omega^{\prime} \in C_{i},-N+1 \leqq i \leqq 0$, it holds $\omega^{\prime}(-1)=-i$, and hence

$$
V\left(\omega^{\prime}\right)=\log \beta_{-i} \text {. }
$$

From Lemma 3-1,

$$
\begin{aligned}
& \left(\varphi\left(\omega^{\prime}\right)\right)(0)=i, \quad\left(\varphi\left(\omega^{\prime}\right)\right)(1)=j, \quad \text { for some } j, \\
& U\left(\varphi\left(\omega^{\prime}\right)\right)=\log \beta_{-i} .
\end{aligned}
$$

3) In case $\omega^{\prime} \in C_{1}$, it holds $\omega^{\prime}(-1)=N$, and hence

$$
I^{r}\left(\omega^{\prime}\right)=\log \beta_{N} .
$$

and

$$
\begin{aligned}
& \left(\varphi\left(\omega^{\prime}\right)\right)(0)=i, \quad\left(\varphi\left(\omega^{\prime}\right)\right)(1)=j, \quad-N+1 \leqq j \leqq 0, \\
& U\left(\varphi\left(()^{\prime}\right)\right)=\log \beta_{Y} .
\end{aligned}
$$

This implies that

$$
U\left(\varphi\left(\omega^{\prime}\right)\right)=V\left(\omega^{\prime}\right), \quad \mu_{\beta} \text {-a.e. }
$$

'Therefore (2) of the Main Lemma is satisfied for $h\left(\omega^{\prime}\right)=0, \quad \omega^{\prime} \in X_{\beta}$. To prove (4) of the Main Lemma, put $\mu_{\beta}{ }^{\prime}=\mu_{\beta} \cdot \varphi^{-1}$, then 
(*) $\sum_{x \in I_{m}{ }^{n}} \mu_{\beta}^{\prime}([x]) U_{n}^{m}(x)$

$$
\begin{aligned}
& =\sum_{x, y \in I_{m^{\prime}}} \mu_{\beta}^{\prime}([x, y]) U(x, y) \\
& +\sum_{i=1}^{n-2} \frac{(i+1)(n-1-i)}{n-1} \sum_{x, y \in I_{m^{\prime}}} \mu_{\beta}^{\prime}\left([x, \underbrace{m^{*}, \cdots, m^{*}}_{i}, y]\right) \\
& \times U_{i+2}^{m}(x, \underbrace{m^{*}, \cdots, m^{*}}_{i}, y) \\
& +\sum_{i=1}^{n-1} \frac{i}{n-1} \sum_{x \in I_{m^{\prime}}}\left[\mu_{\beta}^{\prime}(x, \underbrace{m^{*}, \cdots, m^{*}}_{i}]\right) U_{i+1}^{m}(x, \underbrace{m^{*}, \cdots, m^{*}}_{i}) \\
& \left.+\mu_{\beta}^{\prime}\left([\underbrace{m^{*}, \cdots, m^{*}}_{i}, x]\right) U_{i+1}^{m}(\underbrace{m^{*}, \cdots, m^{*}}_{i}, x)\right] \\
& +\mu_{\beta}^{\prime}\left([\underbrace{m^{*}, \cdots, m^{*}}_{n}]\right) U_{n}^{m}(\underbrace{m^{*}, \cdots, m^{*}}_{n}) \text {. }
\end{aligned}
$$

On the support of $\mu_{\beta}{ }^{\prime}$

$$
U(x, y) \leqq \log \beta_{\max }
$$

where $\beta_{\max }=\max \left\{\beta_{0}, \beta_{1}, \cdots, \beta_{N}\right\} . \quad$ For $i \geqq 1$ we get

$$
\begin{aligned}
& \left|U_{i+2}^{m}(x, \underbrace{m^{*}, \cdots, m^{*}}_{i}, m)\right|=\frac{1}{i+1}\left[U(x, m+i)+\sum_{j=0}^{i-1} U(m+j+1, m+j)\right] \\
& \leqq \log \beta_{\max } \\
& \left|U_{i+1}^{m}(x, \underbrace{m^{*}, \cdots, m^{*}}_{i})\right| \\
& =-\frac{1}{i} \log \sum_{j \geqq 1} r_{m+j} \exp [-U(x, m+i+j) \\
& \left.-\sum_{k=1}^{i-1} U(m+k+j+1, m+k+j)\right] \leqq \log \beta_{m a x}-\frac{1}{i} \log \frac{\beta_{m \alpha x}^{-m}}{1-\beta_{\max }}, \\
& \left|U_{i+1}^{m}(\underbrace{m^{*}, \cdots, m^{*}}_{i}, m)\right| \\
& =-\frac{1}{i} \log \frac{l_{m+i}}{\sum_{j} l_{j} r_{j}} \exp \left[-\sum_{k=0}^{i-1} U(m+k+1, m+k)\right] \\
& \leqq \log \beta_{\text {max }}+\left|\frac{1}{i} \log \frac{l_{m+i}}{\sum_{j} l_{j} r_{j}}\right|, \\
& \left|U_{i+1}^{m}(\underbrace{m^{*}, \cdots, m^{*}}_{i+1})\right|
\end{aligned}
$$




$$
\begin{aligned}
& =\left|-\frac{1}{i} \log \sum_{j \geqq 1} \frac{l_{m+j+i+1} r_{m+j}}{\sum_{k} l_{k} r_{k}} \exp \left[-\sum_{k=1}^{i} U(m+k+j+1, m+k+j)\right]\right| \\
& \leqq \log \beta_{m a x}+\left|\frac{1}{i} \log \frac{\sum_{j \geqq 1} l_{m+j+i+1} r_{m+j}}{\sum_{k} l_{k} r_{k}}\right| .
\end{aligned}
$$

Moreover for $j \geqq 1$

$$
\begin{aligned}
& l_{j}= T^{j} 1 \\
&= \sum_{k=0}^{a_{j}(1)-1} \beta_{k}^{-1}+\beta_{a_{j}(1)}^{-1}\left(\sum_{k=0}^{a_{j+1}(1)-1} \beta_{k}^{-1}\right)+\cdots \\
&=\left\{\beta_{a_{0}(1)}^{-1} \cdots \beta_{a_{j-1}(1)}^{-1} \sum_{k=0}^{a_{j}(1)-1} \beta_{k}^{-1}+\beta_{a_{0}(1)}^{-1} \cdots \beta_{a_{j}(1)}^{-1} \sum_{k=0}^{a_{j+1}(1)-1} \beta_{k}^{-1}+\cdots\right\} \\
& \quad \times \beta_{a_{0}(1)} \cdots \beta_{a_{j-1}(1)} \\
& \geqq C \cdot \mu_{\beta}^{\prime}([j]) \beta_{a_{0}(1)} \cdots \beta_{a_{j-1}(1)},
\end{aligned}
$$

for some constant $C$, and

$$
\iota_{\beta}^{\prime}([k]) \leqq K \beta_{m i n}^{-k}
$$

for some constants $K, k \geqq 1$ and $\beta_{\min }=\min \left\{\beta_{0}, \cdots, \beta_{N}\right\}$. Therefore the right-hand terms of $(*)$ become

$\varlimsup_{m \rightarrow \infty} \varlimsup_{n \rightarrow \infty}$ (the first term) $=\sum_{x, y \in I} \mu_{\beta}{ }^{\prime}([x, y]) U(x, y)=\int d \mu_{\beta}{ }^{\prime} \cdot U$,

$\varlimsup_{m \rightarrow \infty} \varlimsup_{n \rightarrow \infty} \mid$ the second term $\mid$

$$
\begin{aligned}
& \left.\leqq \lim _{m \rightarrow \infty} \sum_{i=1}^{\infty}(i+1) \mu_{\beta}^{\prime}(\underbrace{\left[m^{*}, \cdots, m^{*}\right.}_{i}, m]\right) \log \beta_{\text {max }} \\
& \leqq \lim _{m \rightarrow \infty} \sum_{i=1}^{\infty}(i+1) K \beta_{m i n}^{-m-i} \log \beta_{\text {max }}=0
\end{aligned}
$$

$\varlimsup_{m \rightarrow \infty} \varlimsup_{n \rightarrow \infty} \mid$ the third term $\mid$

$$
\begin{aligned}
& \left.\leqq \varlimsup_{m \rightarrow \infty} \max _{i} \mu_{\beta}^{\prime}(\underbrace{\left[m^{*}, \cdots, m^{*}\right.}_{i}]\right)\left(i \log \beta_{m a x}-\log \frac{\beta_{\max }^{-m}}{1-\beta_{\max }}\right) \\
& \leqq \varlimsup_{m \rightarrow \infty} \max _{i} \sum_{k=m+1}^{\infty} K \beta_{\min }^{-k}\left(i \log \beta_{m a x}-\log \frac{\beta_{\max }^{-m}}{1-\beta_{\max }}\right)=0
\end{aligned}
$$

$\varlimsup_{m \rightarrow \infty} \varlimsup_{n \rightarrow \infty} \mid$ the fourth term $\mid$ 


$$
\begin{aligned}
& \left.\leqq \varlimsup_{m \rightarrow \infty} \max _{i} \mu_{\beta}^{\prime}(\underbrace{\left[m^{*}, \cdots, m^{*}\right.}_{i}, m]\right)\left(i \log \beta_{m a x}+\left|\log \frac{\mu_{\beta}^{\prime}([m+i])}{\sum_{j} l_{j} r_{j}}\right|\right) \\
& =\varlimsup_{m \rightarrow \infty}\left|\max _{i} \mu_{\beta}^{\prime}([m+i]) \log \mu_{\beta}^{\prime}([m+i])\right|=0,
\end{aligned}
$$

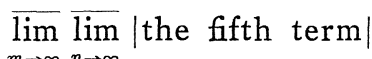

$$
\begin{aligned}
& \leqq \varlimsup_{m \rightarrow \infty} \varlimsup_{n \rightarrow \infty}\left(\sum_{k \geqq m+n+1} \mu_{\beta}^{\prime}([k])\left(\log \beta_{m a x}+\left|\frac{1}{n-1} \log \frac{\sum_{s \geqq 1} l_{m+s+n} r_{m+s}}{\sum_{j} l_{j} r_{j}}\right|\right)\right. \\
& \leqq \varlimsup_{m \rightarrow \infty} \varlimsup_{n \rightarrow \infty} \frac{1}{n-1}\left(\sum_{k \sum_{m+n}} \mu_{\beta}^{\prime}([k])\right)\left|\log \left(\sum_{s \geqq 1} \mu_{\beta}^{\prime}([m+s+n])\right)\right|=0 .
\end{aligned}
$$

Thus (4) of the Main Lemma is proved. Therefore, applying the Main Lemma, we complete the proof of Theorem 2 .

\section{References}

[1] Adler, R. L. and Weiss, B., Simularity of automorphisms of the torus, Mem. A. M. S., No. 98 (1970).

[2] Ito, Sh. and Takahashi, Y., Markov subshifts and realization of $\beta$-expansion, (to appear).

[3] Parry, W., Intrinsic Markov chains, Trans. A. M. S., 112 (1964), 55-66.

[4] - On Rohlin's formula for entropy, Acta Math. Acad. Sci. Hungar., 15 (1964), 107-113.

[5] Pinsker, M. S., Information and informations stability of random variables and processes, Holden Day, Inc., 1964, San Francisco.

[6] Shiokawa, I., Ergodic properties of piecewise linear transformations, Proc. Japan Acad., 46 (1970), 1122-1125.

[7] Spitzer, F., Random fields and interacting particles system, Notes of lectures given at the MAA Summer seminar, Williamstown, Mass., (1971).

[8] Takahashi, Y., Isomorphisms of $\beta$-expansions to Markov automorphisms, Osaka J. Math., 10 (1973), 175-184. 